\title{
Spectral statistics of Toeplitz matrices
}

\author{
Eugene Bogomolny \\ Universit Paris-Saclay, CNRS, LPTMS, 91405 Orsay, France
}

(Dated: July 2, 2020)

\begin{abstract}
Spectral statistics of hermitian random Toeplitz matrices with independent identically distributed elements is investigated numerically. It is found that the eigenvalue statistics of complex Toeplitz matrices is surprisingly well approximated by the semi-Poisson distribution belonging to intermediate-type statistics observed in certain pseudo-integrable billiards. The origin of intermediate behaviour could be attributed to the fact that Fourier transformed random Toeplitz matrices have the same slow decay outside the main diagonal as critical random matrix ensembles. The statistical properties of the full spectrum of real random Toeplitz matrices with i.i.d. elements are close to the Poisson distribution but each of their constituted sub-spectra is again well described by the semi-Poisson distribution. The findings open new perspective in intermediate statistics.
\end{abstract}


Random matrices in physics firstly appeared as a tool for simplified description of complicated (and mostly unknown) quantum Hamiltonian of heavy nuclei [1]. Later the idea of statistical characterisation of physical and mathematical systems with complex behaviour becomes ubiquitous and has been successfully applied to a huge variety of different problems ranging from chaotic systems [2] and number theory [3] to quantum gravity [4], 5]. Physical applications were accompanied by quick increase of mathematical results in random matrix theory (RMT). Besides many others it is worth to mention the exact calculations of eigenvalue distributions for invariant random matrix ensembles [6] and the proof of their universality for wider classes of matrices [7].

One of the oldest class of matrices being investigated is Toeplitz matrices for which matrix elements $T_{m n}$ depend only on the difference of indices $m-n$

$$
T_{m n}=a_{m-n}, \quad m, n=1, \ldots, N
$$

for a certain vector $a_{t}$ with $t=-(N-1), \ldots,(N-1)$.

These matrices arise naturally in many branches of mathematics and physics, such as differential and integral equations, functional analysis, probability theory, numerical analysis, theory of stationary processes, signal processing, statistics, etc. (see e.g. [8], [9] and references therein). One of the impressive development in this field was the asymptotic calculation of the determinants for different classes of Toeplitz matrices, starting with the classical work of Szegö [10] and resulting in the proof of the Fisher-Hartwig conjecture and its generalisations (see e.g. [11] amongst others).

Random (real symmetric) Toeplitz matrices where $a_{n}$ with $n \geq 0$ are independent identically distributed (i.i.d.) real random variables were introduced in [12] where the question of their mean level density had been posed. In [13], [14] it has been proved that the density of normalised eigenvalues for $N \times N$ real symmetric Toeplitz matrices for i.i.d. $a_{n}$ with zero mean value and unit variance (and finite higher moments) converges in the limit $N \rightarrow \infty$ to a new universal distribution

$$
\lim _{N \rightarrow \infty} \frac{1}{N} \sum_{j=1}^{N} \delta\left(E-\frac{e_{j}}{\sqrt{N}}\right)=\rho(E)
$$

independent on the distribution of $a_{n}$. The form of $\rho(E)$ seems to be intractable analytically. In [15] the behaviour of the largest eigenvalues for the above matrices has been investigated. 
Surprisingly, a very natural and important (from quantum chaos point of view) question about the spectral statistics of random Toeplitz matrices seems not to attract attention. Only in the end of Ref. [14] the authors briefly mentioned that in the limit $N \rightarrow \infty$ the local spacings between adjacent normalised eigenvalues for real symmetric Toeplitz matrices should be Poissonian (as for independent random variables).

The main message of this letter is that the spectral statistics of hermitian complex Toeplitz matrices is of different nature and it is well approximated by the so-called semiPoisson statistics. The latter had been introduced in [16] as describing statistical properties of eigenvalues when only the nearest pairs of levels interact as in RMT. The semi-Poisson statistics is quite special and differs from the Poisson distribution typical for integrable models as well as from the Wigner-Dyson statistics appeared for chaotic systems. It serves usually as a reference point for intermediate statistics observed in certain models. The characteristic features of such statistics are (i) repulsion of eigenvalues at small distances as for usual RMT and (ii) exponential decrease of the nearest-neighbour distribution at large distances as for the Poisson distribution. Such behaviour has been firstly observed in the Anderson model at the point of metal-insulator transition [17] and later in certain pseudo-integrable billiards [18], [19]. For example, the semi-Poisson statistics describes well eigenvalue distribution for a rectangular billiard with the barrier in the centre [20] and also appears as a particular case of spectral statistics of interval-exchange map [21] related with the Lax matrix for an integrable Ruijsenaars-Schneider model [21, 23].

Two types of Toeplitz matrices (1) are considered below: (i) complex hermitian matrices where the real and imaginary parts of $a_{n}$ with $a_{-n}=a_{n}^{*}$ are i.i.d. Gaussian random variables with zero mean and unit variance and (ii) real symmetric matrices where $a_{n}$ with $a_{-n}=a_{n}$ are real i.i.d. Gaussian random variables [24]. For each matrix its eigenvalues, $e_{k}$ and corresponding eigenfunctions, $\Psi_{j}\left(e_{k}\right)$, are calculated numerically from the diagonalisation of Toeplitz matrices

$$
\sum_{n=1}^{N} a_{m-n} \Psi_{n}\left(e_{k}\right)=e_{k} \Psi_{m}\left(e_{k}\right) .
$$

Our conclusions are based on numerical calculations of different spectral correlation functions for these types of matrices. For the semi-Poisson (cf. [16], [32]) and the Poisson distributions they are known analytically and are presented in Table If. The nearest-neighbour distribution $P_{n}(s)$ is the probability that two eigenvalues are separated by interval $s$ and there are 
exactly $n$ eigenvalues inside this interval. The expressions in Table $\mathbb{I}$ correspond to the unfolded spectrum normalised to unit density, $s=\bar{d}\left(e_{j+1+n}-e_{j}\right)$ where $e_{j}$ is an ordered set of eigenvalues and $\bar{d}$ is the mean density. Another characteristic quantity indicated in that Table is the two-point correlation formfactor (the Fourier transform of the two-point correlation function) which can conveniently be calculated from the unfolded spectrum by the expression

$$
K(\tau)=\lim _{N \rightarrow \infty}\left\langle\frac{1}{N}\left|\sum_{j=1}^{N} e^{2 \pi i \bar{d} e_{j} \tau}\right|^{2}\right\rangle
$$

where the average is taken over different realisations of the random matrix and eigenvalues in a small window.

To avoid the unfolding it is convenient to calculate the probability distribution of ratio of 3 consecutive eigenvalues $r=\left(e_{j+2}-e_{j+1}\right) /\left(e_{j+1}-e_{j}\right)$ [33. The expected formulas for this distribution are presented in the third row of Table $\mathrm{I}$.

The results of calculations are summarised at Figs. 1, 2 for complex hermitian Toeplitz matrices and at Figs. 3, 4 for real symmetric matrices. In each plot two sets of data are superposed. One corresponds to $200 \times 200$ matrices averaged over 10000 realisations. The second is the result of calculations for $1000 \times 1000$ matrices averaged over 1000 realisations. Only minor changes were observed with increasing the matrix dimensions and/or the number of realisations.

At Fig. 1 the comparison between the first three nearest-neighbour distributions calculated numerically for complex hermitian Toplitz matrices and the semi-Poisson formulas is presented. In calculations only $60 \%$ of eigenvalues around the maximum of the spectral density were taken into account and the spectrum was unfolded by using a third degree polynomial. The agreement is quite good and it seems that with increasing of matrix dimensions the observed distributions are slightly closer to the semi-Poisson ones (see Insert in this figure). At Fig. 2 the results of calculations of the probability distribution of ratio of three nearest eigenvalues and the two-point formfactor for complex matrices are depicted. The agreement with the semi-Poisson formulas is evident. In particular, the level compressibility, $\chi=\lim _{\tau \rightarrow 0} K(\tau)$, is close to 0.5 which is the characteristic value for the semi-Poisson distribution [34]. All obtained results clearly indicate that spectral correlation functions for complex Toeplitz matrices are quite well described by the semi-Poisson predictions.

At Figs. 3, 4 the same correlation functions but for real symmetric Toeplitz matrices are 


\begin{tabular}{lll}
\hline \hline $\begin{array}{l}\text { Correlation } \\
\text { functions }\end{array}$ & $\begin{array}{l}\text { Semi-Poisson } \\
\text { distribution }\end{array}$ & Poisson distribution \\
\hline$P_{n}(s)$ & $\frac{2^{2(n+1)}}{(2 n+1) !} s^{2 n+1} e^{-2 s}$ & $\frac{s^{n}}{n !} e^{-s}$ \\
\hline$K(\tau)$ & $\frac{2+\pi^{2} \tau^{2}}{4+\pi^{2} \tau^{2}}$ & 1 \\
\hline$P(r)$ & $\frac{6 r}{(1+r)^{4}}$ & $\frac{1}{1+r^{2}}$ \\
\hline \hline
\end{tabular}

TABLE I. Main correlation functions for the semi-Poisson and the Poisson distributions

plotted. In this case the results are indeed close to the Poisson predictions as was conjectured in [14] though small deviations are visible. To see clearly the size of these deviations the nearest-neighbour and the ratio distributions were fitted by ad hoc expressions indicated in figure captions.

These figures correspond to statistics of the full spectrum of real Toeplitz matrices (1) as it has been considered in [14]. But it is known [25 27] that due to the built-in symmetry: $T_{N-n+1, N-m+1}=T_{n, m}$ eigenvectors of real symmetric Toeplitz matrices are split into two sub-classes, symmetric and skew-symmetric for which $\Psi_{N-m+1}^{( \pm)}= \pm \Psi_{m}^{( \pm)}$respectively. Consequently the spectrum of real Toeplitz matrices breaks into two sub-spectra with symmetric and skew-symmetric eigenvectors [28]. The sub-spectra can be directly calculated from the diagonalisation of matrices $T^{( \pm)}$of half-dimension [25 27] with $m, n=1, \ldots,[N / 2]$. For even $N$

$$
T^{( \pm)}=\left(a_{m-n} \pm a_{m+n-1}\right)
$$

For odd $N$

$$
\begin{aligned}
& T^{(-)}=\left(a_{m-n}-a_{m+n}\right), \\
& T^{(+)}=\left(\begin{array}{cc}
a_{0} & \sqrt{2} a_{n} \\
\sqrt{2} a_{m} & a_{m-n}+a_{m+n}
\end{array}\right) .
\end{aligned}
$$

Numerical calculations (not shown) demonstrate that the spectral correlation functions of the both sub-spectra are also well described by the semi-Poisson statistics and are practically indistinguishable from the ones of complex Toeplitz matrices indicated at Figs. 1, 2] [30].

In addition to spectral statistics of eigenvalues of Toeplitz matrices it is of importance to investigate statistical properties of their eigenfunctions. In usual ensembles of random matrices eigenfunctions are invariant over rotations [6] and in almost all coordinates they 


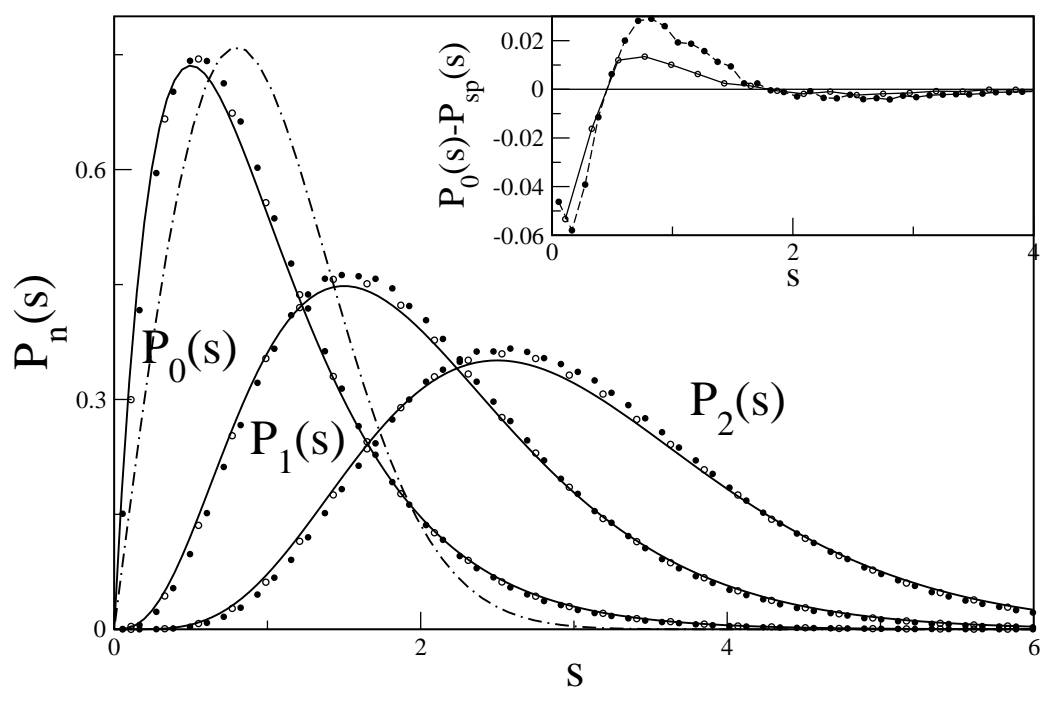

FIG. 1. Three nearest-neighbour distributions for complex hermitian Toeplitz matrices. Filled circles are results for $200 \times 200$ matrices and 10000 realisations. Open circles are data for $1000 \times 1000$ matrices and 1000 realisations. The solid lines indicate the corresponding semi-Poisson formulas. Dashed-dotted line is the Wigner surmise for the nearest-neighbour distribution for the usual Gaussian orthogonal ensemble: $P_{W}(s)=\frac{\pi}{2} s \exp \left(-\frac{\pi}{4} s^{2}\right)$. Insert: the difference between the nearestneighbour distribution computed numerically and the semi-Poisson formula: $P_{\mathrm{sp}}(s)=4 s \exp (-2 s)$.

are fully extended. This is not the case for non-invariant models. Eigenfunctions of complex Toeplitz matrices (and for each sub-spectra for real matrices) in coordinate space given by (3) look as fully extended. From experiences with models with intermediate statistics [35], [36] it follows that the same eigenfunctions in the momentum representation are completely different and they may and will have non-trivial fractal dimensions. The later are defined from the scaling of eigenfunction moments with matrix dimensions (see e.g. [35])

$$
M_{q}=\left\langle\sum_{n=1}^{N}\left|\Psi_{n}(e)\right|^{2 q}\right\rangle \underset{N \rightarrow \infty}{\longrightarrow} C N^{-\tau(q)} .
$$

Here $\Psi_{j}(e)$ is the eigenfunction corresponded to an eigenvalue $e$ (assumed normalised) and the average is taken over a small energy window and different realisation of random matrices. The exponent, $\tau(q)$, defines the fractal dimension: $D_{q}=\tau(q) /(q-1)$. Physically fractal 


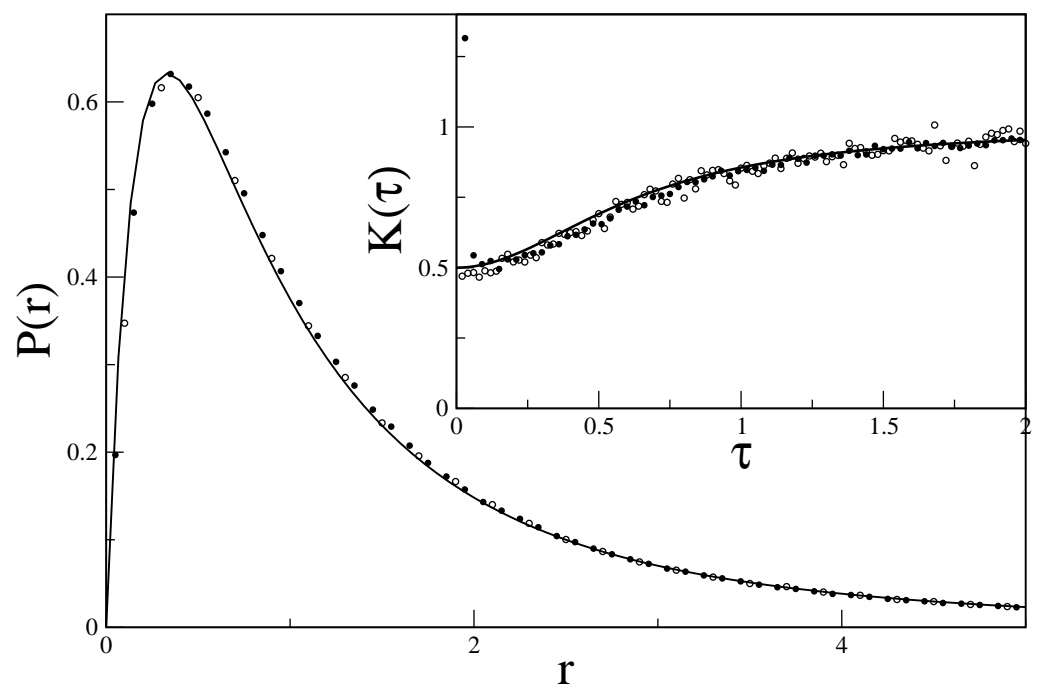

FIG. 2. Comparison between the numerically calculated probability distribution of the ratio of 3 consecutive eigenvalues and the semi-Poisson prediction. Insert: Comparison between the two-point formfactor and the semi-Poisson prediction.

dimensions determine how many important components have eigenfunctions in a certain scale. For fully extended eigenfunctions $D_{q}=1$ and for localised eigenfunctions $D_{q}=0$. The case when $D_{q}$ depends on $q$ corresponds to multifractal eigenfunctions.

For Toeplitz matrices (1) eigenfunctions in the momentum representation can be calculated either by the direct Fourier transform of eigenfunctions of (1)

$$
\hat{\Psi}_{p}(e)=\frac{1}{\sqrt{N}} \sum_{j=1}^{N} e^{2 \pi i p j / N} \Psi_{j}(e)
$$

or by the diagonalisation of the hermitian Toeplitz matrix in the momentum representation

$$
\begin{aligned}
\hat{M}_{p r} & =\frac{1}{N} \sum_{m=1}^{N} \sum_{n=1}^{N} a_{m-n} e^{2 \pi i(m p-n r) / N} \\
& =\xi_{p} \delta_{p r}+\left(1-\delta_{p r}\right) \frac{2 i\left(\eta_{p}-\eta_{r}\right)}{N\left(e^{-2 \pi i(p-r) / N}-1\right)}
\end{aligned}
$$




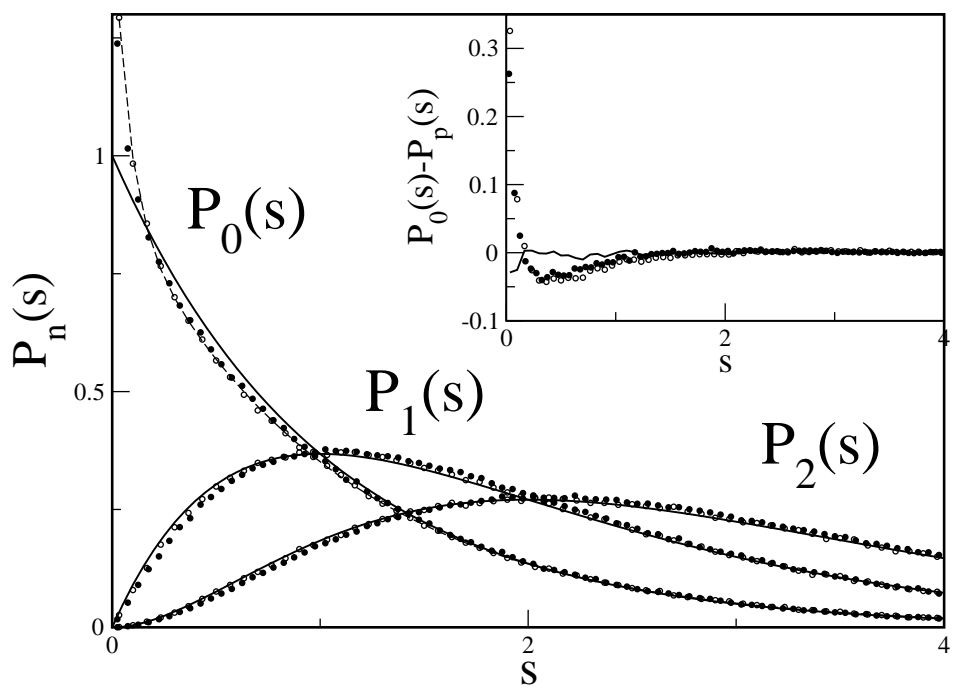

FIG. 3. The same as at Fig. 1 but for real symmetric Toeplitz matrices. Solid lines indicate the Poisson predictions to the considered quantities. Dashed line indicates a fit $P_{\text {fit }}(s)=$ $0.92 e^{-0.96 s}+0.68 e^{-13.7 x}$ to the nearest-neighbour distribution. Insert shows the difference between the numerical nearest-neighbour distribution and the Poisson prediction: $P_{p}(s)=\exp (-s)$. Solid line in the Insert is the difference between the numerics and the above fitting formula.

where

$$
\begin{aligned}
& \xi_{p}=a_{0}+2 \sum_{t=1}^{N-1}\left(1-\frac{t}{N}\right) \operatorname{Re}\left(a_{t} e^{2 \pi i t p / N}\right), \\
& \eta_{p}=\sum_{t=1}^{N-1} \operatorname{Im}\left(a_{t} e^{2 \pi i t p / N}\right) .
\end{aligned}
$$

Numerical determination of fractal dimensions for the Fourier eigenfunctions (8) were performed by the diagonalisation of Toeplitz matrices of dimensions $N=2^{n}$ with $n=7,8,9,10$ averaged over 1000 realisations. Only eigenfunctions with eigenvalues between $-2 \sqrt{N}$ and $2 \sqrt{N}$ for complex matrices and between $-\sqrt{N}$ and $\sqrt{N}$ for real matrices were taken into account. Linear fits in the logarithmic scale work well and permit to determine fractal dimensions. Preliminary results suggest that fractal dimensions are non-trivial: $D_{1 / 2} \approx 0.6$, $D_{3 / 2} \approx 0.5, D_{2} \approx 0.2$ with estimated error of order of 0.1 Larger-scale calculations should be performed to determine precise values of fractal dimensions for Toeplitz matrices. 


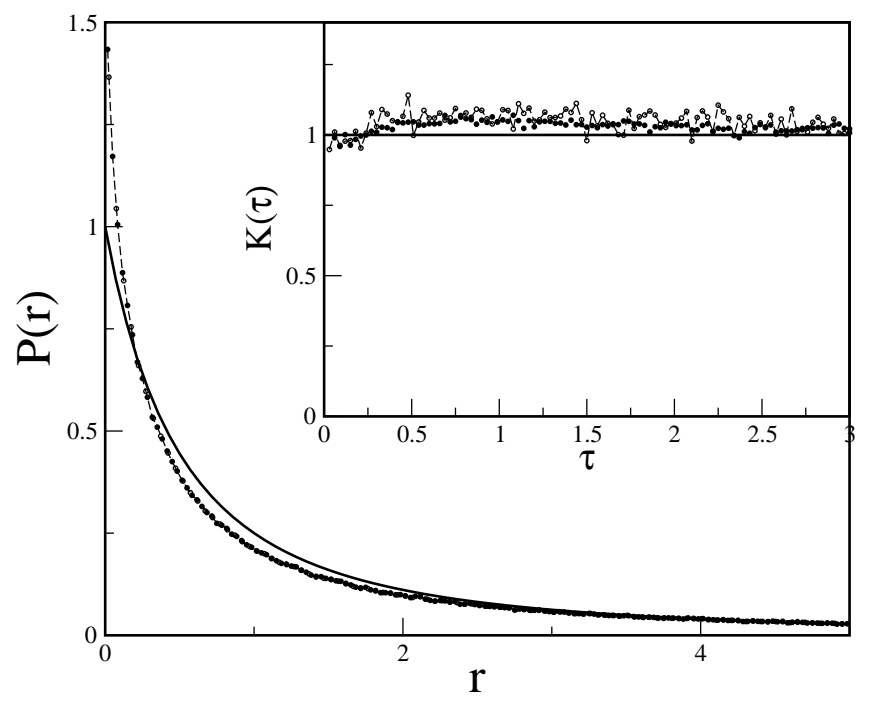

FIG. 4. The same as in Fig. 2 but for real symmetric Toeplitz matrices. Dashed line indicates a fit $P_{\text {fit }}(r)=1.51\left(1+5.44 r+0.90 r^{2}\right)^{-1}$ to the data.

As $\operatorname{Re}\left(a_{n}\right)$ and $\operatorname{Im}\left(a_{n}\right)$ are i.i.d. Gaussian variables with zero mean and unit variance matrix elements $(10)$ and $(11)$ are also Gaussian variables with zero mean and the following variances

$$
\begin{aligned}
\left\langle\eta_{p} \eta_{r}\right\rangle & =N \delta_{p r}-1 \\
\left\langle\xi_{p} \eta_{r}\right\rangle & =\left(1-\delta_{p r}\right) \cot \left(\frac{\pi(r-p)}{N}\right) \\
\left\langle\xi_{p} \xi_{r}\right\rangle & =-1+\delta_{p r} \frac{4 N^{2}+2}{3 N}+\frac{2\left(1-\delta_{p r}\right)}{N \sin ^{2}\left(\frac{\pi(p-r)}{N}\right)} .
\end{aligned}
$$

In [37] an ensemble of power law banded random matrices has been investigated. In this ensemble each matrix element $H_{i j}$ is independent (up to the hermitian symmetry) Gaussian variable with zero mean and variance decreasing as a power from the diagonal; $\left\langle H_{p r}^{2}\right\rangle \sim(p-$ $r)^{-2 \alpha} p, r \gg 1$. It was argued in [37] that when $\alpha>1$ off-diagonal terms are non-essential and the spectral statistics is the same as that of diagonal elements (i.e. Poissonian). When $\alpha<1$ the spectral statistics is that of usual random matrix ensembles. Value $\alpha=1$ is special (see also [38]) and corresponds to the so-called critical ensembles characterised by intermediate spectral statistics similar (but in general not equal) to the semi-Poisson distribution and by 
non-trivial fractal dimensions calculated either numerically or in perturbation series [35, 36].

A characteristic property of matrix $\hat{M}_{p r}$ in $(9)$ is a linear decrease of matrix elements from the main diagonal, $\hat{M}_{p r} \sim\left(\eta_{p}-\eta_{r}\right) /(p-r)$ for $p, r \ll N$ which is precisely the condition of criticality (and of unusual intermediate-type spectral statistics) in random matrix ensembles [38], [37]. Strictly speaking matrix (9) does not belong to the class of matrices discussed in [38], [37]. But physical arguments in these papers are quite general and robust and their conclusions seems to be applicable in more general setting [36].

It is important to stress that the property of slow fall-off of matrix elements of Toeplitz matrices in the Fourier space is valid only because $\eta_{p}$ in 12 does not decrease for large $p \ll N$ and $N \rightarrow \infty$. But $\eta_{p}$ is the Fourier transform of $a_{t}$. For any reasonably smooth function $a_{t}$ its high Fourier harmonics should go to zero and matrix (9) would be not critical. Consequently, its spectral statistics should be the close to the statistics of the diagonal part.

For total spectrum of real random Toeplitz matrices (without the explicit splitting into two sub-spectra (5), (6) ) such arguments do not work as their diagonal matrix elements (10) are doubly degenerated $\xi_{p}=\xi_{N-p}$ (with $\eta_{p}=-\eta_{N-p}$ ) and the off-diagonal terms serve mostly to remove this degeneracy. Such degenerate case was not investigated within critical banded matrix approach. Numerical results at Figs. 3 and 4 suggest that a certain tendency of gluing levels together remains at least for finite $N$.

The main result of this letter is the demonstration that spectral statistics of complex Toeplitz matrices is unusual and in the case of i.i.d. Gaussian elements is surprisingly well described by the semi-Poisson distribution. The root of such intermediate type statistics is the closeness of Fourier transformed Toeplitz matrices to critical random matrix ensembles characterised by a linear decrease of matrix elements from the main diagonal. In turn, this property is related with non-convergence of random Toeplitz matrix symbols. Such conditions are quite robust and should be valid for different random Toeplitz matrices but they cannot explain the observed closeness of eigenvalue statistics of complex Toeplitz matrices with i.i.d. matrix elements to the semi-Poisson distribution. In the absence of analytical results the semi-Poisson distribution should be considered as a kind of simple Wigner-type surmise which approximate well different spectral correlation functions of Toeplitz matrices.

The case of real Toeplitz matrices is special. Statistics of their full spectrum resembles the Poisson distribution though for relatively moderate matrix dimensions small deviations were observed. Nevertheless, spectral statistics of each of two sub-spectra for these matrices 
is close to the semi-Poisson distribution.

The intermediate statistics till now appeared only in certain (rare) non-generic models. The facts that (i) Toeplitz matrices appear naturally in very different fields and (ii) their statistical properties are of intermediate type indicate that intermediate statistics in general and the semi-Poisson distribution in particular are more universal that was considered before. Further investigation of these phenomena is of considerable interest.

The author is greatly indebted to O. Giraud for pointing out Ref. [27] and numerous useful discussions.

[1] E. Wigner, Ann. Math. 62, 548 (1955); SIAM Review, 9,1 (1967).

[2] O. Bohigas, M.-J. Giannoni, and C. Schmit, Phys. Rev. Lett. 52, 1 (1984).

[3] J.P. Keating and N.C. Snaith, Comm. Math. Phys. 214, 57 (2000).

[4] S. Sachdev and J. Ye, Phys. Rev. Lett. 70, 3339 (1993).

[5] A. Kitaev, http://online.kitp.ucsb.edu/online/entangled15/ (2015).

[6] M.L. Mehta Random matrices, Academic Press Inc. 3rd ed. (2004).

[7] T. Tao and V. Vu, Acta Math. 206, 127 (2011).

[8] A. Böttcher and B. Silbermann, Introduction to Large Truncated Toeplitz Matrices, Springer New York (1999).

[9] U. Grenander and G. Szegö, Toeplitz forms and their applications, Univ. of California Press, Berkeley, Los Angeles (1958).

[10] G. Szegö, Orthogonal polynomials, Amer.Math. Soc., Providence, R.I. (1959).

[11] P. Deift, A. Its, and I. Krasovsky, Ann. Math. 174, 1243 (2011); Comm. Pure Appl. Math. 66, 1360 (2013).

[12] D. Bai, Statistica Sinica, 611 (1999).

[13] W. Bryc, A. Dembo, and T. Jiang, Ann. Prob. 34, 1 (2006).

[14] C. Hammond and S. J. Miller, J. Theor. Probab. 18, 537 (2005).

[15] A. Sen and B. Virág, Ann. Prob. 41, 4050 (2013).

[16] E. Bogomolny, U. Gerland, and C. Schmit, Eur. Phys. J. B 19, 121 (2001).

[17] B. I. Shklovskii et al., Phys. Rev. B 47, 11487 (1993).

[18] P.J. Richens and M.V.Berry, Physica D, 2, 495 (1981). 
[19] E. B. Bogomolny, U. Gerland, and C. Schmit, Phys. Rev. E 59, 1315 (1999).

[20] J. Wiersig, Phys. Rev. E 65, 046217 (2002).

[21] E. Bogomolny and C. Schmit, Phys. Rev. Lett., 93, 254102 (2004).

[22] E. Bogomolny, O. Giraud, and C. Schmit, Phys. Rev. Lett. 103, 054103 (2009).

[23] E. Bogomolny, O. Giraud, and C. Schmit, Nonlinearity, 24, 3179 (2011).

[24] For pure imaginary hermitian Toeplitz matrices with $a_{t}=i b_{t}$ and $b_{t}(t=1, \ldots, N-1)$ being real i.i.d. Gaussian variables the results are practically the same as for complex matrices discussed in the text.

[25] A. L. Andrew, Linear Algebra Appl. 7, 151 (1973).

[26] A. Cantoni and F. Butler, Linear Algebra Appl. 13, 275 (1976).

[27] W. F. Trench, Notes of the real symmetric Toeplitz eigenvalues problem, (1994), http://works.bepress.com/william_trench/136/.

[28] For complex hermitian Toeplitz matrices anti-unitary symmetry: $T_{N-n+1, N-m+1}=T_{n, m}^{*}$ implies that their eigenvectors can be chosen as follows: $\Psi_{N-m+1}=\Psi_{m}^{*}$. The existence of this anti-unitary symmetry forces the complex random Toeplitz matrices to have linear level repulsion [29].

[29] M. Robnik and M. V. Berry, J. Phys. A: Math. Gen. 19,669 (1986).

[30] The two sub-spectra are not exactly interlacing and the construction of [31] can not be applied.

[31] H. Hernández-Saldaña, J. Flores, and T. H. Seligman, Phys. Rev. E 60, 449 (1999).

[32] Y. Y. Atas et al., J. Phys. A: Math. Theor., 46, 355204 (2013).

[33] V. Oganesyan and D. A. Huse, Phys. Rev. B 75, 155111 (2007).

[34] Deviations of numerally calculated formfactor at very small $\tau$ from a $N$-independent expression are expected as formally $K(0)=N$ (see (4) ) and an increasingly large number of realisations is required to perform correctly the averaging at very small $\tau$.

[35] F. Evers, A. D. Mirlin, Rev. Mod. Phys. 80, 1355 (2008).

[36] E. Bogomolny and O. Giraud, Phys. Rev. E 84, 036212 (2011); Phys. Rev. E 85, 046208 (2012).

[37] A. D. Mirlin et al., Phys. Rev. E 54 , 3221 (1996).

[38] L. S. Levitov, Phys. Rev. Lett. 64, 547 (1990). 